\title{
Comparison of Data Reduction Methods for the Analysis of Iyashi Expressions using Brain Signals
}

\author{
Julian Romero $^{1,2}$, Luis Diago ${ }^{1,2}$, Junichi Shinoda ${ }^{1,2}$, Ichiro Hagiwara ${ }^{* 2}$ \\ ${ }^{1}$ InterLocus, Inc. Japan \\ ${ }^{2}$ Meiji Institute for Advanced Study of Mathematical Sciences, Meiji University, Japan \\ ${ }^{*}$ ihagi@meiji.ac.jp
}

Received: October 17, 2014; Accepted: December 25, 2014; Published: December 24, 2015

\begin{abstract}
Recent advances in simulation in science and engineering are focusing on automatic measuring of human-feelings about a product design by using Kansei Engineering words. Iyashi is a Japanese word used to describe a peculiar phenomenonthat is mentally soothing, but is yet to be clearly defined. This paper explores the analysis of Electro Encephalogram (EEG) brain signals (Alpha, Beta) as a method to determine when a stimulus (face images) generates Iyashi in a person and produces good feelings. NeuroSky B3 Headband is used as a device to obtain the EEG signals and Holographic Neural Networks (HNN) are evaluated to predict the level of Iyashi. Nine data dimensionality reduction algorithms for brain signals are explored to improve the level of prediction of the HNN. The experimental results show that the percent of prediction with $\mathrm{HNN}$ can be increased in more than $10 \%$ if data reduction methods are applied to Beta brain waves.
\end{abstract}

Keywords: Iyashi, Electroencephalogram, Brain signal analysis, NeuroSky, Holographic Neural networks, Data Reduction Algorithms.

\section{Introduction}

Kansei Engineering originated in Japan and it maps individual features of a product to customer's subjective responses. Collection of customer data is a key component of Kansei Engineering; consumers evaluate a set of products to decide how much a design evokes each Kansei word (e.g. stylish, elegant, and exciting). In Japan, Iyashi is a popular buzzword today, referring to anything that is physically or mentally soothing [1,2]. Iyashi goods- books, music, pictures, incense and aromas, bath salts, and plants - abound, offering to heal the physical and 
Journal of Advanced Simulation in Science and Engineering

psychological stress of the workplace and of daily life in general [2]. Although there are instances where by the English word Healing is used to explain the Japanese word Iyashi, it is not appropriate since there are healthy people who also consume Iyashi products [1]. In society the expression Iyashi-kei is frequently used to describe lay person who simply help people to relax.

In this paper we focus on the level of Iyashi that is generated to a person during an experiment where visual stimuli are presented to the subject. There are many different ways to analyze emotion using a computer. Some researches show that humans can recognize emotions from voice and faces with $70-98 \%$ of accuracy and computer can classify facial expression with about $80-90 \%$. However it has to be noted that these results are obtained on very controlled circumstances, and could differ in ordinary situations $[3,4]$. On the other hand, emotions are not always displayed on a facial expression. In psychology, an explicit separation is made between the physiological arousal, the behavioral expression (affect), and the conscious experience of an emotion (feeling). Facial expression and voice can be consciously adapted, and its interpretation is not objective. For this reason, research has been conducted to look for other physiological aspects that are more difficult to self-modify like the user's heart rate, skin conductance, and pupil dilation $[3,4,5]$.

In the past decades, pioneer researchers have tried to apply EEG data analysis techniques developed in electrical engineering and information theory, including time/frequency analysis and Independent Component Analysis (ICA) [6,7]. These techniques have revealed EEG processes whose dynamic characteristics are also correlated with behavioral changes. For example, short-term changes in spectral properties of the ongoing EEG in specific frequency bands may be correlated with cognitive processes, e.g. expectancy of a target stimulus and with visual awareness. To date, the majority of Brain-Computer Interface (BCI) systems rely on EEG recordings. In this paper, NeuroSky's B3 Band was evaluated as a minimally invasive method to measurebrain signal behavior (Alpha, Beta) of a subject during aevaluation of a group of portraits showing Iyashi expressions $[1,12]$. Also different algorithms to reduce data dimensionality were evaluated in order to improve the level of prediction of Holographic Neural Networks (HNN).

The rest of this paper is organized as follows. Section 2 includes a brief description about EEG brain signals and the NeuroSky B3 band. The experimental montage and the HNN used in this paper are also reviewed. Section 3 shows the data dimensionality reduction methods explored in this paper for the prediction of iyashi expressions from brain signals. In section 4, the experimental methods, materials and results of prediction by different data dimensionality reduction methods using HNN are presented and compared. The results are discussed in Section 5 and the main conclusions and future works are presented in Section 6. 


\section{Journal of Advanced Simulation in Science and Engineering}

\section{Related Works}

EEG signals are obtained with electrodes located on the top of the head using the 10/20 electrode position standard shown in Figure 1.

\subsection{EEG features}

The use of this topographic system and the analysis of frequencies are important to identify the different cerebral rhythms behavior. The first one indicates that each one of the cerebral activities have a specific distribution in the skull and the second one indicates that each of the different cerebral rhythms adopt a specific band of frequency. The analysis of these frequencies is the first step to identify the brain behavior. These frequencies are descripted by Greek letters: Alpha $(8-12 \mathrm{~Hz})$,Beta $(12-30 \mathrm{~Hz})$, Theta $(4-7.5 \mathrm{~Hz})$, Gamma (more than $32 \mathrm{~Hz})$, and Delta (Less than $4 \mathrm{~Hz})[4,8]$.

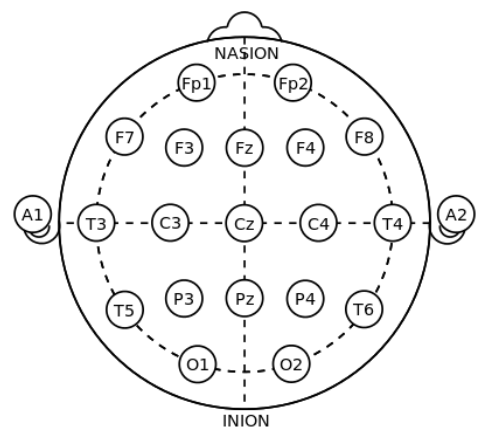

Figure 1:10-20 electrode placement system.

If a person is awaken, lay, and with the eyes closed the main brain activity is in the Alpha band frequency. This particular frequency has a major activity in the occipital region of the skull, where, depending of the age of the person can oscillate between 50-120 mV. However, this brain frequency can be reduced or blocked when the persons open their eyes or they are influenced by auditory stimuli. The Alpha frequency is important to know when a person is with the eyes closed but awaken. It's usually related with a state of relaxness [8].

The second brain frequency to be analyzed is Beta. This brain activity possesses a low voltage behavior $(15-50 \mathrm{mV})$. This frequency has a major neural activity in the frontal and pre-frontal areas of the skull. The presence of this type of frequency is related with the rational decision, concentration, state of decision making, and complex calculation $[8,9]$.

The Theta brain signal is considered a moderate low voltage activity that is no more than $100 \mathrm{mV}$. It is uniformly distributed in all the regions of the skull and it has major amplitude in the frontal-center-temporal region with bilateral behavior. It is related with the transition between relaxness to sleep and it has presence in deep sleep states (state I and state II) [8].

The Gamma brain signal is consider a fast frequency activity with very low voltage behavior (less than $5 \mathrm{mV}$ ). We can consider that there is Gamma activity when two or more different senses are active (e.g., Sight, hear, or touch among others) at the same time or when the 


\section{Journal of Advanced Simulation in Science and Engineering}

person uses a short-time memory matching of recognized objects, sounds, or tactile sensations.

The Delta brain signal occurs mainly when a person is in a very deep sleep state (state III and state IV) and has a very low presence in the awake state. The main use of the Delta frequency is to establish how deep a person is sleeping [8].

Previous researches have found that the major source of information related with emotional behavior can be found in the Frontal-Parietal area of the skull, and that the frequencies with more information are the Alpha and Beta because these two signals are more related with the attention and relaxation levels of a person $[4,9,10]$. In this paper, Iyashi levels perceived by a group of subjects are predicted from their Alpha and Beta waves.

\subsection{NeuroSky B3 Headband}

NeuroSky Inc. has developed a non-invasive, dry, biosensor to read electrical activity in the brain. NeuroSky is a low-cost, easy to use EEG developed for leisure. It captures neural activity using three dry electrodes (locations: beneath the ears and the forehead) and decodes them by applying some algorithms. NeuroSky provides information on a user's Delta, Theta, Alpha, Beta, and Gamma brainwave band power levels. eSense is a NeuroSky's proprietary algorithm for characterizing mental states. To calculate eSense values, the Neuro Sky thinkGear technology amplifies the raw brainwave signal and removes the ambient noise and muscle movement. The eSense algorithm is then applied to the remaining signal, resulting in the interpreted eSense meter values. Note that eSense meter values do not describe an exact number, but instead describe ranges of activity.

In previous researches, EEG systems have been used as a way to measure emotional feelings [4,17]. In [4], BraInquary PET 2.0 EEG device was used to acquire signals from the F3, F4, and Fpz of the 10/20 allocation system. Using this device, the Alpha and Beta waves were analyzed in order to determinate if these two signals have some relationship with emotional feelings evoked from visual and auditory stimuli selected from the International Affective Digitized Sound system (IADS) and the International Affective Picture System (IAPS). Instead of using the IADS/IAPS we focuses on the analysis of Iyashi expressions which cannot be perceived form above databases (See details in [12]). The research in [17] is also closely related with this paper. Biological signals such as electrocardiogram (ECG), galvanic skin reflex (GSR), breathing rate, and EEG were measured while watching "Kawaii" stimulus. "Kawaii" is also a Japanese word that represents a kansei value; it has positive meanings, such as cute, lovable, and small. "Kawaii" maybe related with Iyashi but its relationship is out of the scope of this paper. In [17], the authors focused on color and size of "kawaii" objects and showed that heart rate increases while watching "kawaii" stimuli. Although the au- 


\section{Journal of Advanced Simulation in Science and Engineering}

thors mention that the human mental state is more active while watching "kawaii" objects, no result is shown in the paper about the classification of mental states from brain waves. Hence this paper is focused on the classification of Iyashi expressions by using EEG raw signals recorded from Fp1 and Fp2 instead of F3 and F4. Instead of using NeuroSky B3 band to analyze eSense (i.e. Attention, Meditation) levels as in our previous research [11], in this paper the Alpha and Beta waves are used for classification.

\subsection{Experimental montage}

Most of the emotion recognition systems in previous research are based on the difference in hemispherical activity in the frontal cortex. This difference can be recorded using bipolar electrode measurements crossing the hemispheres. This means the ground electrode will be connected to a neutral site (e.g., left or right ear), while two electrode are available for recording bipolar signals. The most-used locations for EEG-based emotion recognition are Fp1 and Fp2 (e.g. NeuroSky Headband) because these positions are above the prefrontal area, with as a large advantage that it is easy to apply, and there is no hair growth on the forehead [4].

A software that acquires EEG signals was designed using NeuroSky B3 headband while some portraits are shown to the tested subject (see Figure 2). During the experiment, the subjects used the keyboard to rate each stimulus on the scale '1' - YES, '2' - I don't know, and ' 3 ' NO, to express whether or not they feel a Iyashi stimulus. A total of 20 images with different expressions were used. Nationality, gender, and age range (divided in decades) of the subjects are also registered. Different from our previous work [12], in this paper the subjects do not have limited time to answer in order to obtain a large amount of EEG data from the evaluation of each picture.

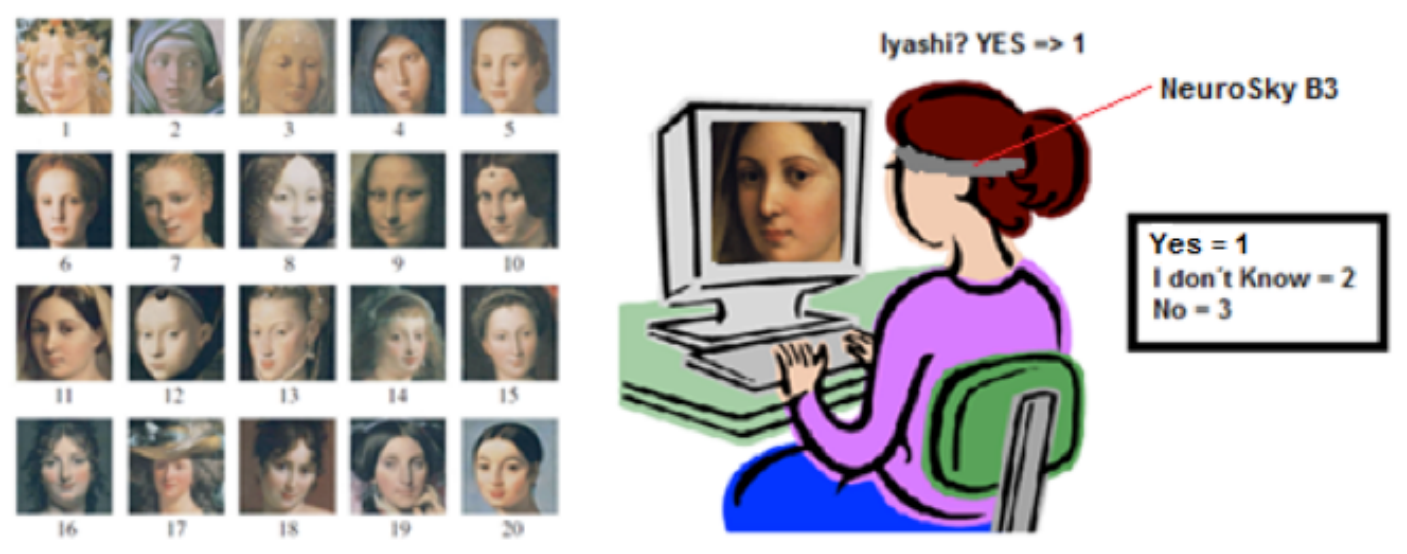

Figure 2:Left: Images Data set. Right: Experimental Setup [1,12]. 


\section{Journal of Advanced Simulation in Science and Engineering}

\subsection{Holographic Neural Networks}

This paper is continuation of a previous work [12] in which we introduced fuzzy quantification to build a MF describing how subjects classify Iyashi-stimulus into fuzzy groups. Compared with former HNN and SVM classifiers, the proposed combination of HNN with FQ-II archived the best classification accuracy using less training iterations than SVM classifiers (see [12] and reference therein for details). However, there were ambiguous cases where the subject himself could not decide the state that has been induced by the system. Sparked in parts by the growing interest in the evaluation of multiple communication channels to determine the inner states of a person, we combine the facial expressions of the subject with signals from the brain to clarify the state induced on the person in ambiguous situations. The extraction of cognitive state information from neural signals will produce appropriate feedback to the system and it will increase the classification accuracy.

\subsection{Validation method for HNN}

In order to give a baseline for comparison, leave-one-out cross-validation (LOOCV) method was tested for classification with HNN using features from three different frequency configurations (Alpha only, Beta only, and the combination of Alpha and Beta). In the LOOCV method, an image from the database is eliminated and the classifier is trained to reproduce the associations with the remaining images. After training, the classifier is tested for the prediction of the removed image. This process is repeated for all the images in the database. After this, each of the results obtained with the HNN and the real answer given by the user are evaluated [12].

\section{Data Dimensionality Reduction Methods}

An important part to improve the performance of the HNN is to reduce the number of EEG features to be analyzed. There are many methods to reduce the data dimensionality [15]. Feature selection algorithms used to reduce data dimensionality are divided into filters, wrappers, and embedded approaches. In this paper a wrapper approach is used. Wrappers utilize the HNN to score subsets of inputs according to their predictive power. The following explains the methods used in this paper for the selection of the subsets of inputs.

\subsection{Principal Components Analysis (PCA)}

PCA is a linear technique for dimensionality reduction, which means that it performs a transformation from a group of data with many elements in a linear subspace to a lower dimensionality data. PCA constructs a low-dimensional representation of the data that describes as much of the variance in the data as possible. This is done by finding a linear basis of reduced dimensionality for the data, in which the amount of variance in the data is maximal. 


\section{Journal of Advanced Simulation in Science and Engineering}

In mathematical terms, PCA attempts to find a linear mapping $\mathbf{M}$ that maximizes the cost function trace $\left(\mathbf{M}^{T} \operatorname{cov}(\mathbf{X}) \mathbf{M}\right)$, where $\operatorname{cov}(\mathbf{X})$ is the sample covariance matrix of the data X. It canbeshown that this linear mapping is formed by the $d$ principal eigenvectors (i.e., principal components) of the sample covariance matrix of the zero-mean data. Hence, PCA solves the eigenproblem

$$
\operatorname{cov}(\boldsymbol{X}) \boldsymbol{M}=\lambda \boldsymbol{M} .
$$

The eigenproblem is solved for the $d$ principal eigenvalues $\lambda$. The low-dimensional data representations $\boldsymbol{y}_{\boldsymbol{i}}$ of the datapoints $\boldsymbol{x}_{\boldsymbol{i}}$ are computed by mapping them onto the linear basis $\mathbf{M}$, i.e., $\mathbf{Y}=\mathbf{X M}$.

PCA is identical to the traditional technique for multidimensional scaling (MDS) called classical scaling. The input into classical scaling is, likethe input into most other multidimensional scaling techniques, a pairwise Euclidean distance matrix $\mathbf{D}$ whose entries $\boldsymbol{d}_{i j}$ represent the Euclidean distance between the high-dimensional datapoints $\boldsymbol{x}_{\boldsymbol{i}}$ and $\boldsymbol{x}_{\boldsymbol{j}}$. Classical scaling finds the linear mapping $\mathbf{M}$ that minimizes the cost function

$$
\phi(\boldsymbol{Y})=\sum_{i j}\left(d_{i j}^{2}-\left\|\boldsymbol{y}_{\boldsymbol{i}}-\boldsymbol{y}_{j}\right\|^{2}\right) .
$$

In which $\left\|\boldsymbol{y}_{\boldsymbol{i}}-\boldsymbol{y}_{\boldsymbol{j}}\right\|^{2}$ is the squared Euclidean distance between the low-dimensional datapoints $y i$ and $\boldsymbol{y}_{j}, \boldsymbol{y}_{i}$ is restricted to be $\boldsymbol{x}_{\boldsymbol{i}} \mathbf{M}$, and $\left\|m_{j}\right\|^{2}=1$ for $\forall_{j}$. It can be shown that the minimum of this cost function is given by the eigendecomposition of the Gram matrix $\mathbf{K}=\mathbf{X X}^{\boldsymbol{T}}$ of the high-dimensional data. The entries of the Gram matrix can be obtained by double-centering the pairwise squared Euclidean distance matrix, i.e., by computing

$$
k_{i j}=\frac{1}{2}\left(d_{i j}^{2}-\frac{1}{n} \sum_{l} d_{i l}^{2}-\frac{1}{n} \sum_{l} d_{j l}^{2}+\frac{1}{n^{2}} \sum_{l m} d_{l m}^{2}\right) .
$$

The minimum of the cost function in (2) can now be obtained by multiplying the principal eigenvectors of the double-centered squared Euclidean distance matrix (i.e., the principal eigenvectors of the Gram matrix) with the square-root of their corresponding eigenvalues [13].

\subsection{Probabilistic PCA}

PCA may also be viewed upon as a latent variable model called probabilistic PCA (PPCA). This model uses a Gaussian prior over the latent space, and a linear-Gaussian noise model. The probabilistic formulation of PCA leads to an EM-algorithm that may be computationallymore efficient for very high-dimensional data. By using Gaussian processes, probabilistic PCA may also be extended to learn nonlinear mappings between the high-dimensional and the low-dimensional space. Another extension of PCA also includes minor components (i.e., the eigenvectors corresponding to the smallest eigenvalues) in the linear mapping, as minor components may be of relevance in classification setting [13]. 


\section{Journal of Advanced Simulation in Science and Engineering}

\subsection{LLC and Factor Analysis (FA)}

Locally Linear Coordination methods (LLC) compute a number of locally linear models and subsequently performs a global alignment of the linear models. This process consists of two steps: 1 - computing a mixture of local linear models on the data by means of an EM-algorithm and 2- aligning the local linear models in order to obtain the low-dimensional data representation using a variant of Local Linear Embedding (LLE).

LLC first create a mixture of $m$ factor analyzers (that is a mixture of Gaussians model with a restriction on the covariance of the Gaussians) using the EM algorithms. Alternatively, a mixture of probabilistic PCA model could be employed. The local linear models in the mixture are used to construct $\mathrm{m}$ data representations $\boldsymbol{z}_{\boldsymbol{i j}}$, and their corresponding responsibilities $\boldsymbol{r}_{i j}$ (where $j \in\{1, \ldots, m\}$ ) for every datapoint $\boldsymbol{x}_{\boldsymbol{i}}$. The responsibilities $\boldsymbol{r}_{\boldsymbol{i j}}$ describe to what extent datapoint $\boldsymbol{x}_{\boldsymbol{i}}$ corresponds to the model $\boldsymbol{j}$; they satisfy $\sum_{j} r_{i j}=1$. Using the local models and the corresponding responsibilities, responsibility-weighted data representations $\boldsymbol{u}_{\boldsymbol{i j}}=$ $\boldsymbol{r}_{\boldsymbol{i j}} \boldsymbol{z}_{\boldsymbol{i j}}$ are computed. The responsibility-weighted data representations $\boldsymbol{u}_{\boldsymbol{i j}}$ are stored in a $n x$ $m D$ block matrix $\mathbf{U}$. The alignment of the local models is performed based on $\mathbf{U}$ and on a matrix $\mathbf{M}$ that is given by $\boldsymbol{M}=\left(\boldsymbol{I}_{\boldsymbol{n}}-\boldsymbol{W}\right)^{\boldsymbol{T}}\left(\boldsymbol{I}_{\boldsymbol{n}}-\boldsymbol{W}\right)$. Heirein, the matrix $\mathbf{W}$ contains the reconstruction weights computed by LLE, and $\boldsymbol{I}_{\boldsymbol{n}}$ denotes the $n \times n$ identify matrix. LLC aligns the local models by solving the generalized eigenproblem

$$
A v=\lambda B v,
$$

for the $d$ smallest nonzero eigenvalues. In (4), $\mathrm{A}$ is the inproduct of $\mathbf{M}^{T} \mathbf{U}$ and $\mathrm{B}$ is the inproduct of $\mathbf{U}$. The d eigenvectors $\boldsymbol{v}_{\boldsymbol{i}}$ form a matrix $\mathbf{L}$ that defines a linear maping from the reponsibility-weighted data representation $\mathbf{U}$ to theunderlying low-dimensional date representation $\mathbf{Y}$. The low-dimensional data representation is thus obtained by computing $\mathbf{Y}=\mathbf{U L}$ [13].

\subsection{Sammon's Mapping}

This algorithm adapts the classical scaling cost function (2) by weighting the contribution of each pair $(i, j)$ to the cost function by the inverse of their pairwise distance in the high-dimensional space $d_{i j}$. In this way, the cost function assigns roughly equal weight to retaining each of the pairwise distances, and thus retains the local structure of the data better than classical scaling. Mathematically, the Sammon cost function is given by

$$
\phi(Y)=\frac{1}{\sum_{i j} d_{i j}} \sum_{i \neq j} \frac{\left(d_{i j}-\left\|y_{i}-y_{j}\right\|^{2}\right)}{d_{i j}},
$$

where $d_{i j}$ represents the pairwise Euclidean distance between the high-dimensional data points $\boldsymbol{x}_{\boldsymbol{i}}$ and $\boldsymbol{x}_{\boldsymbol{j}}$, and the constant in front is added in order to simplify the gradient of the costfunction. 


\section{Journal of Advanced Simulation in Science and Engineering}

\subsection{Kernel PCA and GPLVM}

Kernel PCA (KPCA) is a reformulation of traditional linear PCA in a high-dimensional space that is constructed using a kernel function. KPCA computes the principal eigenvectors of the kernel matrix, rather than those of the covariance matrix. The reformulation of PCA in kernel space is straightforward, since a kernel matrix is similar to the inner product of the datapoints in the high-dimensional space that is constructed using the kernel function. The application of PCA in the kernel space provides KPCA the property of constructing nonlinear mapping.

KPCA computes the kernel matrix $\mathrm{K}$ of the datapoints $x_{i}$. The entries in the kernel matrix are defined by

$$
k_{i j}=K\left(x_{i}, x_{j}\right) \text {, }
$$

where $K$ is a kernel function, which may be any function that gives rise to a positive-semidefinite kernel K. Subsequently, the kernel matrix $\mathbf{K}$ is double-centered using the following modification of the entries

$$
k_{i j}=\frac{1}{2}\left(k_{i j}-\frac{1}{n} \sum_{l} k_{i l}-\frac{1}{n} \sum_{l} k_{j l}+\frac{1}{n^{2}} \sum_{l m} k_{l m}\right) .
$$

The centering operation corresponds to subtracting the mean of the features in traditional PCA: it subtracts the mean of the data in the features space defined by the kernel function $K$. As a result, the data in the features space defined by the kernel function is zero-mean. Subsequently, the principal $d$ eigenvectors $v_{i}$, of the centered kernel matrix are computed. The eigenvectors of the covariance matrix $a_{i}$ (in the feature space constructed by $K$ ) can now be computed, since they are related to the eigenvectors of the kernel matrix $v_{i}$ through

$$
a_{i}=\frac{1}{\sqrt{\lambda_{i}}} v_{i}
$$

In order to obtain the low-dimensional data representation, the data is projected onto the eigenvectors of the covariance matrix $a_{i}$. The result of the projection (i.e., the low-dimensional data representation $\mathbf{Y}$ ) is given by

$$
y_{i}=\left\{\sum_{j=1}^{n} a_{1}^{(j)} K\left(x_{j}, x_{i}\right), \ldots, \sum_{j=1}^{n} a_{d}^{(j)} K\left(x_{j}, x_{i}\right)\right\},
$$

where $a_{1}^{(j)}$ indicates the $j$ th value in the vector $a_{1}$ and $K$ is the kernel function that was also used in the computation of the kernel matrix.

Like KPCA, the Gaussian Process Latent Variable Model (GPLVM) also uses kernel functions to construct non-linear variants of probabilistic PCA. However, the GPLVM is not simply the probabilistic counterpart of KPCA: in the GPLVM, the kernel function is defined over the low-dimensional latent space, whereas in KPCA, the kernel function is defined over the high-dimensional data space [13]. 


\section{Journal of Advanced Simulation in Science and Engineering}

\section{Experimental Results}

This section shows the experimental methods, materials, and the evaluation of the subjects analyzed on this paper.

\subsection{Materials, subjects and testing procedures}

The tests were performed using an ASUS ${ }^{\circledR}$ X53SD Laptop with Windows ${ }^{\circledR} 7$ OS, and programmed using the API from NeuroSky Inc. ${ }^{\circledR}[14]$ in Microsoft Visual $\mathrm{C}++{ }^{\circledR}$ 2013. The NeuroSky B3 headband was used as EEG recording system. This headband use two dry electrode recorders localized in Fp1 and Fp2 of the 10/20 system, and its reference could be allocated in $\mathrm{A} 1$ or $\mathrm{A} 2$ (One of the ears). A1 is used in all experiments to normalize the results and always have the same source of noise.

In advance, the subject was instructed about the experiment. Brief information about the nature and the meaning of the kansei word Iyashi was explained for non-Japanese people. The word Iyashi was explained by using other English words like relief, well-being, confortable or satisfaction. The Iyashi expression was defined as follows:"The expression gives you the impression as if the holder seems to accept you exactly now and talk to you. And you want to reveal your own true emotion instinctively". Then 20 pictures are shown to the subject, one by one. Answers and EEG data are saved on a text file if the user presses a key ('1', '2 'or ' 3 ') depending on the level of Iyashi produced by the image.

The number of subjects that participated in the test is 6 , each one with varying gender, age and nationality. The information of each subject can be appreciated in the following Table 1.

Table 1. Information of the tested subjects.

\begin{tabular}{llll}
\hline Subject & Gender & Age & Nationality \\
\hline A & Male & $20-29$ & Colombian \\
\hline B & Male & $40-49$ & Cuban \\
C & Male & $20-29$ & French \\
D & Female & $20-29$ & Colombian \\
E & Female & $50-59$ & Japanese \\
F & Male & $50-59$ & Japanese \\
\hline
\end{tabular}

\subsection{Data Acquisition and analysis}

Multi-threating process (MTP) has been programed to acquire a huge amount of data for the analysis. Two threads have been used, the first thread was responsible of showing images to the user, and the other for data acquisition from the NeuroSky B3 band taking samples with a rate of $512 \mathrm{~Hz}$. The system was programed using de Application Programming Interface (API) provided by NeuroSky Inc. to control de functions of the headband. 
Journal of Advanced Simulation in Science and Engineering

As it was explained in section 2.1, the frequencies with the most number of emotion information are the Alpha $(8-12 \mathrm{~Hz})$ and Beta $(12-30 \mathrm{~Hz})$. Frequency bandpass filters were used to extract each of these two regions from the raw EEG signal in order to analyze the information of each of these signals. Extracting only the region of interest we can avoid other frequencies (noise) that could affect the analysis. The influence of involuntary movements like eye blinking are more dominant below $4 \mathrm{~Hz}$, Electro Cardiogram (ECG) or heart beats around $1.2 \mathrm{~Hz}$, and muscle movements above $30 \mathrm{~Hz}$. Nonphysiological artifacts influence caused by electromagnetic devices or power lines are above $50 \mathrm{~Hz}$ [4]. For this paper, the bandpass filter implementation provided by EEGLab for Matlab ${ }^{\circledR}$ was used.

For each one of the Stimuli, 1000 samples from the EEG (Alpha and Beta) are used. In previous projects the Bayesian Networks (BN) and Fisher's Discriminant analysis (FDA) have already been applied for classifying EEG signals into different classes of emotions [4]. In this paper HNN are used for classification of subject's answers about Iyashi instead of BN or FDA. The answers for each one of the subjects A, B, C, D, E and F can be observed in the following Table 2 .

Table 2: Answers provided by the subjects about Iyashi levels in 20 images.

\begin{tabular}{llllllllllllllllllllll}
\hline Subject & 1110 \\
\hline & $\underline{\mathbf{1}}$ & $\underline{\mathbf{2}}$ & $\underline{\mathbf{3}}$ & $\underline{\mathbf{4}}$ & $\underline{\mathbf{5}}$ & $\underline{\mathbf{6}}$ & $\underline{\mathbf{7}}$ & $\underline{\mathbf{8}}$ & $\underline{\mathbf{9}}$ & $\underline{\mathbf{1 0}}$ & $\underline{\mathbf{1 1}}$ & $\underline{\mathbf{1 2}}$ & $\underline{\mathbf{1 3}}$ & $\underline{\mathbf{1 4}}$ & $\underline{\mathbf{1 5}}$ & $\underline{\mathbf{1 6}}$ & $\underline{\mathbf{1 7}}$ & $\underline{\mathbf{1 8}}$ & $\underline{\mathbf{1 9}}$ & $\underline{\mathbf{2 0}}$ \\
$\mathbf{A}$ & 2 & 2 & 3 & 2 & 2 & 3 & 1 & 2 & 1 & 2 & 1 & 2 & 3 & 1 & 1 & 1 & 1 & 1 & 2 & 1 \\
$\mathbf{B}$ & 1 & 1 & 1 & 1 & 2 & 1 & 2 & 1 & 3 & 2 & 3 & 1 & 1 & 2 & 2 & 3 & 2 & 3 & 2 & 3 \\
$\mathbf{C}$ & 1 & 2 & 3 & 3 & 1 & 1 & 1 & 2 & 2 & 3 & 1 & 3 & 3 & 3 & 2 & 3 & 1 & 1 & 2 & 3 \\
$\mathbf{D}$ & 2 & 3 & 1 & 3 & 1 & 1 & 2 & 1 & 1 & 3 & 3 & 2 & 1 & 1 & 1 & 3 & 1 & 3 & 2 & 2 \\
$\mathbf{E}$ & 1 & 1 & 2 & 2 & 2 & 3 & 1 & 3 & 1 & 2 & 1 & 3 & 2 & 2 & 3 & 2 & 3 & 2 & 1 & 1 \\
F & 1 & 1 & 1 & 1 & 2 & 1 & 3 & 1 & 2 & 1 & 3 & 1 & 2 & 2 & 1 & 2 & 3 & 3 & 2 & 2 \\
\hline
\end{tabular}

\section{Degree of Iyashi $\mathrm{N}=6$}

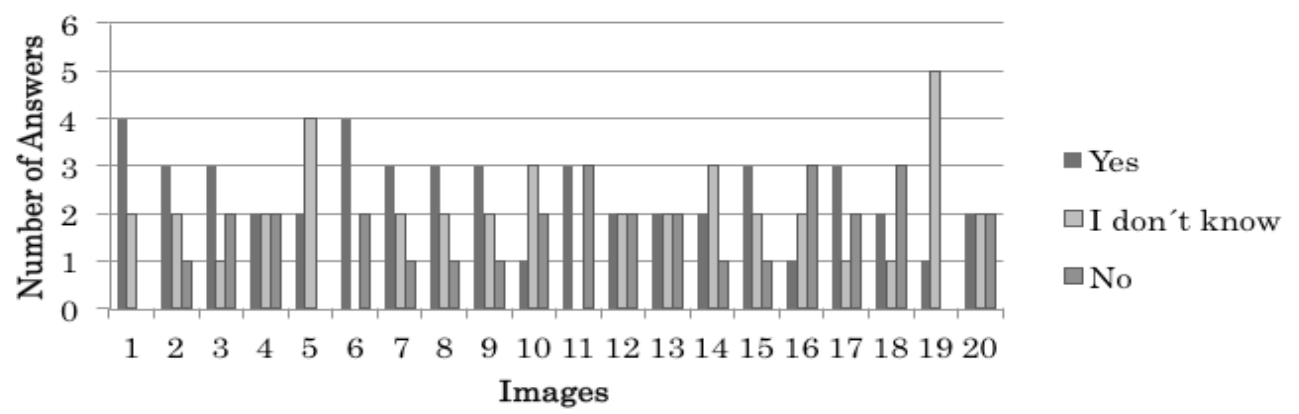

Figure 3: Frequency distribution of Iyashi for each image according to 6 subjects.

In Figure 3 we can observe the total of answers (1-Iyashi, 2-I don't know, 3- No) that each im- 


\section{Journal of Advanced Simulation in Science and Engineering}

age obtains for all 6 participants exposed as a frequency distribution. As we can see each one of the faces have variations on its answers. This is because the grade of perception and definition of Iyashi differ on each person. It is necessary later to develop an exclusive HNN for each one of the subjects in order to do a correct prediction. This result agrees with previous result reported in [12].

\subsection{Comparison of data reduction methods}

Alpha and Beta signals were extracted from the original EEG using a bandpass filter in EEGLab software (one for Alpha $8-12 \mathrm{~Hz}$, and other to Beta $12-30 \mathrm{~Hz}$ ). The program that reads the EEG signal gives a sample when the user presses a key to answer. This sample and other 999 previous samples were taken as the EEG signal to be analyzed in correspondence with the shown picture. We consider that the decision making occurs a few seconds before the moment when the key is pressed (512Hz give us 1000 samples in two seconds approx.).

Alpha and Beta signals are extracted from the raw EEG signal and packed into 1000 samples to train the HNN. Table 3 shows the mean percent of prediction obtained by the LOOCV explained in section 2.5 using raw signals from six subjects. The final row shows the mean and the standard deviation (STD) of the results with all subjects.

Table 3: Mean \% of Prediction using HNN with Raw Signals

\begin{tabular}{llll}
\hline Subject & Alpha only & Beta only & Alpha \& Beta \\
\hline A & 37.5 & 45 & 25 \\
B & 10 & 35 & 30 \\
C & 30 & 20 & 30 \\
D & 55 & 40 & 47.5 \\
E & 35 & 25 & 20 \\
F & 40 & 15 & 35 \\
Mean \pm STD\% & $\mathbf{3 4 . 5 \pm 1 3 . 4}$ & $\mathbf{3 0 \pm \mathbf { 1 0 . 8 }}$ & $\mathbf{3 1 . 2 \pm \mathbf { 8 . 6 }}$ \\
\hline
\end{tabular}

In the Table 3, a mean prediction of $34.5 \%$ was obtained using only Alpha signals as input to the HNN, 30\% using only Beta signals, and 31.2\% using a combination of Alpha and Beta signals. However this percentage can be improved by reducing the number of inputs. Nine data reduction methods were explored by using the Reduction Data Toolbox for Matlab ${ }^{\circledR}$ : Principal Component Analysis (PCA), Probabilistic Component Analysis (PPCA), Classic Multidimensional Scaling (MDS), Lineal Discriminant Analysis (LDA), Factor Analysis (FA), Gaussian Process Latent Variable Model (GPLVM), Sammon's Mapping (Sammon), Neighborhood Components Analysis (NCA), and Maximally Collapsing Metric Learning Algorithm (MCML). The data reduction algorithms were applied to the original Alpha, Beta, and the sum of Alpha plus Beta signals before training the HNN. The number of data on each 


\section{Journal of Advanced Simulation in Science and Engineering}

variable were reduced from 1000 to 20 for all data reduction methods as a baseline for comparisson. The following tables (Table 4-12) show the results of each reduction algorithm.Analyzing all data reduction methods, the percentage of prediction of the HNN was incremented (more than $10 \%$ in some cases) and the level of dispersion (STD) was reduced. However, some of the reducing methods were more stable than others. For example, in Table 4, Table 5 and Table 6 that represent the PCA, PPCA and MDS the average percentage of prediction of the $\mathrm{HNN}$ is near $36 \%$ (more than $4 \%$ increase compared with the average in Table 3 ) but the level of dispersion is higher using Alpha plus Beta (15.7\%).

Table 4: Mean \% of Prediction using HNN with PCA reduction

\begin{tabular}{llll}
\hline Subject & Alpha only & Beta only & Alpha \&Beta \\
\hline A & 50 & 30 & 15 \\
B & 30 & 25 & 50 \\
C & 20 & 30 & 35 \\
D & 35 & 30 & 60 \\
E & 55 & 45 & 20 \\
F & 35 & 35 & 40 \\
Mean \pm STD\% & $\mathbf{3 7 . 5 \pm 1 1 . 8}$ & $\mathbf{3 2 . 5} \pm \mathbf{6 . 3}$ & $\mathbf{3 6 . 6 \pm 1 5 . 7}$ \\
\hline
\end{tabular}

Table 5: Mean \% of Prediction using HNN with Probabilistic PCA reduction.

\begin{tabular}{llll}
\hline Subject & Alpha only & Beta only & Alpha \&Beta \\
\hline A & 35 & 45 & 60 \\
B & 40 & 35 & 15 \\
C & 25 & 35 & 35 \\
D & 25 & 20 & 45 \\
E & 20 & 60 & 30 \\
F & 30 & 60 & 35 \\
Mean \pm STD\% & $29.2 \pm 6.8$ & $\mathbf{4 2 . 5 \pm 1 5 . 7}$ & $\mathbf{3 6 . 6 \pm 9 . 8}$ \\
\hline
\end{tabular}

For LDA (see Table 7) the level of prediction is improved by using samples from Alpha plus Beta (32.5\%). However, the dispersion of the predictions doesn't reduce significantly ( $p$-value $=0.8504$ ) compared with that of using raw signals (see Table 3 ). That is why the use of LDA could be insufficient. 
Journal of Advanced Simulation in Science and Engineering

Table 6: Mean \% of Prediction using HNN with MDS reduction.

\begin{tabular}{|c|c|c|c|}
\hline Subject & Alpha only & Beta only & Alpha \&Beta \\
\hline $\mathbf{A}$ & 50 & 30 & 15 \\
\hline B & 30 & 25 & 50 \\
\hline C & 20 & 30 & 35 \\
\hline D & 35 & 30 & 60 \\
\hline $\mathbf{E}$ & 55 & 45 & 20 \\
\hline $\mathbf{F}$ & 35 & 35 & 40 \\
\hline Mean \pm STD $\%$ & $37.5 \pm 11.8$ & $32.5 \pm 6.3$ & $36.7 \pm 15.7$ \\
\hline
\end{tabular}

Table 7: Mean \% of Prediction using HNN with LDA reduction.

\begin{tabular}{llll}
\hline Subject & Alpha only & Beta only & Alpha \&Beta \\
\hline A & 25 & 40 & 55 \\
B & 5 & 30 & 40 \\
C & 40 & 30 & 20 \\
D & 40 & 25 & 40 \\
E & 35 & 15 & 20 \\
F & 30 & 40 & 20 \\
Mean \pm STD\% & $29.2 \pm 12.0$ & $30 \pm 8.7$ & $\mathbf{3 2 . 5} \pm \mathbf{1 3 . 5}$ \\
\hline
\end{tabular}

In FA (see Table 8), the percentage of prediction also increased if only Beta signals are used ( $7.5 \%$ increase) and the STD is less than $11 \%$ in all the estimations. Also it was the only reduction method that STD decreased as average compared with the results in Table 3.

Table 8: Mean \% of Prediction using HNN with FA reduction.

\begin{tabular}{llll}
\hline Subject & Alpha only & Beta only & Alpha \&Beta \\
\hline A & 40 & 50 & 25 \\
B & 20 & 35 & 20 \\
C & 35 & 45 & 15 \\
D & 25 & 45 & 30 \\
E & 20 & 15 & 35 \\
F & 25 & 35 & 25 \\
Mean \pm STD\% & $27.5 \pm 7.5$ & $\mathbf{3 7 . 5} \pm \mathbf{1 1 . 5}$ & $25 \pm 6.5$ \\
\hline
\end{tabular}

The GPLVM method inTable 9 achieved results similar to that of PPCA. The prediction percent was increased if only Beta (8.3\% increase) and the combination of Alpha \& Beta $(5.4 \%$ increase) are used. 
Journal of Advanced Simulation in Science and Engineering

Table 9: Mean \% of Prediction using HNN withGPLVM reduction.

\begin{tabular}{llll}
\hline Subject & Alpha only & Beta only & Alpha \&Beta \\
\hline A & 45 & 25 & 50 \\
B & 35 & 30 & 35 \\
C & 20 & 15 & 20 \\
D & 35 & 45 & 35 \\
E & 20 & 65 & 35 \\
F & 30 & 50 & 45 \\
Mean \pm STD\% & $30.8 \pm 8.8$ & $\mathbf{3 8 . 3} \pm \mathbf{1 6 . 7}$ & $\mathbf{3 6 . 7 \pm 9 . 4}$ \\
\hline
\end{tabular}

Much better results are obtained with Sammon's Mapping. For some subjects, a high level of prediction (70\%) was obtained (see subject D in Table 10). Using Alpha only, 7.2\% increase in the predictions is achieved (i.e. $41.7 \%)$ but the dispersion is still high $(\sim 14 \%)$.

Table 10: Mean \% of Prediction using HNN withSammon reduction.

\begin{tabular}{llll}
\hline Subject & Alpha only & Beta only & Alpha \& Beta \\
\hline A & 35 & 35 & 40 \\
B & 35 & 20 & 60 \\
C & 40 & 30 & 30 \\
D & 70 & 40 & 20 \\
E & 45 & 35 & 40 \\
F & 25 & 25 & 20 \\
Mean \pm STD\% & $\mathbf{4 1 . 7 \pm 1 4 . 0}$ & $\mathbf{3 0 . 8} \pm \mathbf{6 . 7}$ & $\mathbf{3 5 \pm 1 3 . 8}$ \\
\hline
\end{tabular}

For MCML and NCA in Tables 11 and 12, the prediction percent also increased if only Beta (37.5\%) and the combination of Alpha\&Beta (31.7\%) are used for classification.

Table 11: Mean \% of Prediction using HNN withMCML reduction.

\begin{tabular}{llll}
\hline Subject & Alpha only & Beta only & Alpha \&Beta \\
\hline A & 20 & 30 & 30 \\
B & 10 & 30 & 20 \\
C & 30 & 30 & 20 \\
D & 35 & 40 & 35 \\
E & 30 & 55 & 10 \\
F & 10 & 40 & 60 \\
Mean \pm STD\% & $22.5 \pm 9.9$ & $\mathbf{3 7 . 5} \pm \mathbf{9 . 0}$ & $29.2 \pm 15.9$ \\
\hline
\end{tabular}


Journal of Advanced Simulation in Science and Engineering

Table 12: Mean \% of Prediction using HNN with NCA reduction.

\begin{tabular}{llll}
\hline subject & Alpha only & Beta only & Alpha \&Beta \\
\hline A & 35 & 25 & 30 \\
B & 20 & 20 & 50 \\
C & 45 & 55 & 20 \\
D & 30 & 25 & 25 \\
E & 25 & 20 & 55 \\
F & 30 & 35 & 10 \\
Mean \pm STD\% & $30.8 \pm 7.9$ & $30 \pm 12.2$ & $\mathbf{3 1 . 7 \pm 1 5 . 9}$ \\
\hline
\end{tabular}

\section{Discussions}

According to the above results none of the algorithms outperform the others. However, the percentage of prediction of the HNN was incremented by all data reduction methods(e.g. more than $10 \%$ for PPCA using only Beta signals). A low percent of prediction ( $33 \%$ as average) and a high grade of dispersion for some methods (between 6.3-16.7\%) were still observed in the experimental results. This could be because we only analyzed 6 subjects with 20 images in different environments. All tests were carried out in different places with different conditions of light. The not standardization of the environment can be one of the reasons why the results were affected. For example, two of the tests (C and D) where carried out in a very bright room. This could produce that the subjects have closed their eyes more often, thus incrementing their Alpha signal. The analysis of eye blinking during the experiment by using video camera is currently under development [16]. Removing EEG data acquired during eye blinking could improve the prediction results.

All the images used on the test process where woman's faces. In [4], one of the points of discussion was if the influence of the gender could be a reason of variability in the acquisition of the EEG signal, producing significant changes on Alpha and Beta signals. In the future it could be normalized showing the same number of woman and men's faces. A database with more subjects, standardized environment, and filtered EEG data could provide more information about the performance of proposed algorithms in more details.

The data reduction methods presented in this paper have not been optimized according to number of features to be used for classification. If the data dimensionality is reduced too much, the resulting information could be ambiguous [4,13]. For the reducing process, the group of 1000 samples was reduced to 20 in order to analyze the behavior with all reducing methods applying HNN as a classifier, and provide a baseline for comparison of all data reduction algorithms. 


\section{Journal of Advanced Simulation in Science and Engineering}

\section{Conclusions and Future Works}

Clarifying the meaning of Iyashi from human brain signals could be very interesting for the communities of Kansei engineering and simulation. In this paper an EEG device (NeuroSky B3 band) was evaluated in combination with HNN for predicting the level of Iyashi perceived by a group of subjects during the evaluation of 20 portrait images. Despite different kind of environments, subjects, age, gender and nationality, the level of prediction in some cases can be near $70 \%$. Although the average level of prediction is still very low (30 40\%), the paper showed that an increase of $10 \%$ can be achieved in the prediction percent if Beta signals are used as features for the EEG analysis of Iyashi. Although none of the data reduction algorithms presented in the paper outperform the others, the sammon mapping and factor analysis showed promising results despite the reduced number of input samples.

Our previous work [12] investigated the characteristics of the facial images perceived as Iyashi by one hundred and fourteen subjects (102 Japanese and 12 non-Japanese). In this paper we addressed how to use EEG signals to improve the predictions of Iyashi expressions made by HNN. Currently, we are focusing on non-Japanese in order to clarify the meaning of Iyashi expression considering different nationalities. Nationality differences will be fully addressed in future works. As the HNN was trained without optimal selection of the input features, in our future works we will increase the number of subjects and optimize the feature selection process removing EEG data acquired during eye blinking.

\section{Acknowledgement}

Several parts of the research work were carried out by the support of Grants-in-Aid for scientific research under Grant No. 26420211. We acknowledge its aid dearly.

\section{References}

[1] T. Kitaoka, L. Diago, I. Hagiwara: Definition, detection and generation of Iyashiexpressions, JSME J. Comput. Sci. Technol., 2:4 (2008), 413-422.

[2] T. Matsui: Institutionalization of consumer needs: The case of the 'Healing boom' in Japan, Graduate School of Commerce and Management Center for Japanese Business Sutdies, Hitotsubashi University, Tech. Rep. 071 (2008).

[3] K. Takahashi: Remarks on emotion recognition from bio-potential signals, in Proceedings of the Second International Conference on Autonomous Robots and Agents, New Zealand, 2004, 186-191.

[4] D. O. Bos: EEG-based Emotion Recognition: The Influence of Visual and Auditory Stimuli,Department of Computer Science, University of Twente, Netherlands, 2006. 


\section{Journal of Advanced Simulation in Science and Engineering}

[5] T. Partala, M. Jokiniemi, V. Surakka: Pupillary responses to emotionally provocative stimuli, in Proceedings of the Eye Tracking Research and Application Symposium (ETRA 2000), Florida, 2000, 123-129.

[6] T. Musha, Y. Terasaki, H. A. Haque, G. A. Ivanitsky: Feature extraction from EEGs associated with emotions, Artif Life Robotics, 1 (1997), 15-19.

[7] K. Murakami, M. Nakagawa: On the Emotion Analysis with High-order Correlation Coefficients of NIRS Signals, in Proceedings of the 2nd International Conference on Kansei Engineering and Affective Systems, 1:1 (2008), 311-316.

[8] L. E. Morrillo: Analisis visual del electroencefalograma, Guia Neurologica 7 - Neuro Eledtro Diagnostico, Asociacion Colombiana de Neurologia, 2005, 143-163.

[9] Y. Gil, S. Seo, J. Lee: EEG Analysis of Frontal Lobe Activities by Decision Stimuli, in Proc. 2008 Second International Conference on Future Generation Communication and Networking, Hainan Island, 2008, 13-15.

[10] S. H. Seo, J. T. Lee: Stress and EEG, Convergence and Hybrid Information Technologies, Korea, 2010, 413-426.

[11] J. Romero, L. Diago, I. Shinoda, I. Hagiwara: Kansei Evaluation of Color Differences using Brain Signals,Dynamics and Design Conference 2014, Tokyo, 2014.

[12] L. Diago, T. Kitaoka, I. Hagiwara, T. Kambayashi: Neuro-Fuzzy Quantification of Personal Perceptions of Facial Images Based on a Limited Data Set, IEEE Transactions on Neural Networks, 22:12 (2011), 2422-2434.

[13] L. van der Maaten, E. Postma, J. vn den Herik: Dimensionality reduction: A Comparative Review, Tilburg centre for Creative Computing, (2009), 1-35.

[14] NeuroSky Inc: http://developer.neurosky.com/docs/doku.php?id=app_notes_and_tutorials.

[15] I. Guyon, A. Elisseef: An Introduction to variable and feature selection, J. Machine Learning Research, 3 (2003), 1157-1182.

[16] J. Romero, L. Diago, I. Hagiwara: Verification of Models of Personal Perception of Faces by Closed-eye Classifier using Histogram Correlation, JSST2014, Kitakyushu, 2014.

[17] M. Ohkura, S. Goto, A. Higo, T. Aoto: Relationship between Kawaii Feeling and Biological Signals, Transactions of Japan Society of KanseiEngineerig, 10:2 (2011), 109-114. 